\title{
La evolución del constructivismo (desde una
perspectiva constructivista)
}

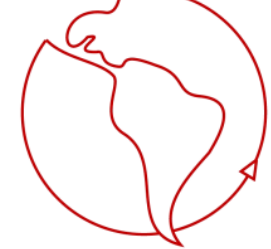

\section{Christian Blanco, Andrés Cabrera, Tomás Gaete \& Juan} Pablo Pinilla

Alumnos del Magíster en Análisis Sistémico Aplicado a la Sociedad. Facultad de Ciencias Sociales, Universidad de Chile mass.facso@uchile.cl

Resumen

El presente artículo reflexiona respecto de las condiciones de evolución del constructivismo en la sociedad, desde la perspectiva de la teoría de los sistemas sociales de Luhmann. Inicialmente se revisan algunas de las nociones básicas de la teoría de la evolución socio-cultural desde el constructivismo del autor, centrándonos en los conceptos de variación, selección y estabilización de semánticas en estructuras de expectativas. Se sugiere que estos elementos encuentran escenarios empíricos de observación en comunicaciones científicas y filosóficas, así como en producciones culturales. La discusión conduce a la pregunta por la probabilidad de re-estabilización del constructivismo en expectativas que operan en la vida cotidiana. Se concluye con la hipótesis de que el constructivismo -en sus expresiones en varios sistemas- constituye una adquisición evolutiva, en tanto permite el acoplamiento de nuevas comunicaciones progresivamente más complejas en la sociedad contemporánea.

\section{Abstract}

This article discusses the evolutionary conditions of constructivism on society, from Luhmann's social systems theory. First, we review some of the basic notions of the theory of social and cultural evolution, focusing on the concepts of variation, selection and stabilization of semantics in expectation structures. We suggest that these elements find empirical scenarios suitable for observation in scientific and philosophical communications, as well as in cultural productions. Our discussion leads to the question regarding the likeliness of stabilization of constructivism in expectations that operate in everyday life. We conclude with the hypothesis that constructivism -on its expressions on several systems- constitutes an evolutionary acquisition, as it allows the coupling of new and more complex communications in contemporary society.

Palabras Clave: Constructivismo, observación, evolución, vida cotidiana, estabilización

Keywords: Constructivism, observation, evolution, everyday life, stabilization

\section{Constructivismo y evolución}

Sobre la base de una epistemología constructivista, Luhmann (1998; 2007) ha propuesto una teoría de la evolución socio-cultural cuyas categorías aplicamos al desarrollo del propio constructivismo, con objeto de indagar en sus condiciones de estabilización en la sociedad contemporánea. Este procedimiento de re-entry del constructivismo es problemático, en tanto abre nuevos escenarios de indagación 
teórica. Por una parte, tal aproximación constituye un espacio para la puesta en juego de la recursividad de la teoría, en la medida que reconoce formas de variación, selección y estabilización en la expresión de las ideas constructivistas. En un segundo momento, abre la posibilidad de observar el desenvolvimiento empírico de comunicaciones constructivistas en el marco del debate científico, filosófico y en la vida cotidiana, constatando manifestaciones de instalación y difusión del constructivismo en la actualidad. Finalmente, es necesario reconocer evidencias que respalden la tesis de la probabilidad de su progresiva estabilización, poniendo en juego categorías constructivistas a disposición de las comunicaciones en la sociedad.

La teoría de la evolución no es original de las ciencias sociales, pero como característicamente ha ocurrido, muchos de los conceptos de las ciencias naturales han sido utilizados para comprender fenómenos sociales, en tanto mecanismos explicativos plausibles (Haines, 2007). El argumento evolucionista -procedente de la biología- se ha usado tanto para la explicación del cambio social, así como también para proponer analogías o metáforas de procesos que se dan en las sociedades humanas. Si bien existen diversas aproximaciones evolucionistas en las ciencias sociales -psicología, antropología, economía, entre otras- procederemos atendiendo al caso de la sociología, específicamente a la teoría luhmanniana de la evolución, pues estimamos que es en esta concepción donde el argumento adquiere mayor grado de generalización y mejores rendimientos heurísticos. La teoría de Luhmann, en efecto, sitúa a la evolución en un estatus epistemológico como mecanismo general aplicable a la sociedad en su conjunto y no sólo a aspectos parciales de la misma, como ocurre en otras aproximaciones.

Luhmann (2007: 355) reconoce así su herencia darwinista, indicando que la sociedad y su estructura son producto de la evolución. En efecto, la existencia de procesos análogos en los sistemas sociales requiere de explicaciones en esta línea. Razeto-Barry y Cienfuegos (2008) han señalado que "la tarea principal de la teoría de la evolución es explicar cómo es posible que algunas estructuras cada vez más improbables [...] surjan y luego funcionen como normales". Proponemos que el constructivismo ha dado señales de emergencia y normalización semántica en diversas áreas de la sociedad, incluidas las esferas científicas, filosóficas y culturales.

En este trabajo sugerimos una vía alternativa de aproximación al constructivismo a partir de los recursos teóricos que entrega esta concepción. Moviéndonos en un nivel de abstracción menor -no en el plano de los "fundamentos operativos" de la teoría sino en el nivel de sus "componentes dinámicos"-, buscaremos desplegar un modelo de interpretación del constructivismo en su propia evolución. Estos elementos dicen relación con "las construcciones teóricas parciales o temáticas que resultan de las operaciones teóricas y que se basan en los supuestos ontológicos y epistemológicos de las mismas" (Mascareño, 2008: 220). No pretendemos aquí dar respuestas respecto del argumento evolutivo luhmanniano, sino operacionalizarlo para interpretar el fenómeno de la emergencia y tentativa consolidación de una semántica constructivista en la sociedad actual.

El artículo desarrolla un abordaje sistémico al constructivismo. Para ello se ensaya una operación de re-entry de la forma constructivista en la teoría, indicando luego su evolución en la dimensión temporal. Se busca sugerir que es posible observar elementos constructivistas en el desarrollo del pensamiento occidental, y su gradual estabilización en la estructura de expectativas que opera en la vida cotidiana. 
El artículo inicia con una exposición de las nociones básicas de la teoría de la evolución socio-cultural desde el constructivismo de Luhmann, centrándose en las nociones de variación, selección y estabilización de semánticas en estructuras de expectativas. En el apartado siguiente estos elementos son evidenciados como expresiones propias del devenir científico y filosófico. Se muestra finalmente cómo es que comunicaciones constructivistas germinan en producciones e ideas que circulan en la sociedad occidental contemporánea. El texto cierra con una problematización de las probabilidades de estabilización del constructivismo en tanto expectativas disponibles en la vida cotidiana.

\section{El constructivismo luhmanniano}

La conceptualización del constructivismo en la obra Niklas Luhmann logra fundamentar epistemológicamente una teoría de los sistemas sociales (Arnold y Robles, 2004) para la comprensión de una sociedad compleja que "ya no se deja reducir por monólogos basados en teorías totalizantes" (Arnold, 2004: 7). De ahí que el énfasis se haya puesto sobre la teoría de la observación, la teoría de las distinciones y la teoría de sistemas de la cibernética como recursos para la comprensión de una sociedad policontextual, donde coexisten distintos planos de descripción y donde conviven diversas racionalidades (Arnold, 2006: 328ss). La incorporación de la evolución como mecanismo general explicativo de la emergencia y formación de estructuras sociales no implica renunciar a esta constatación de policontextualidad social. De hecho, la evolución actúa en el plano operativo de la sociedad, toda vez que sus componentes son indicados desde la dimensión temporal. No obstante, la observación del devenir social no ofrece puntos privilegiados para su descripción.

El constructivismo es la base epistemológica de la teoría de sistemas sociales de Luhmann. Desde la dimensión objetual del sentido, se ha indicado al constructivismo como un mecanismo de auto-reflexión de la sociedad contemporánea, en la medida en que este dispositivo logra desnaturalizar $-y$, por tanto, volver contingente- el orden social. Una vía complementaria es indicar que el constructivismo, remitiéndonos al ámbito de la dimensión social, constituye un programa emergente del sistema científico, que aspira a su generalización en las estructuras que norman la producción y difusión de comunicaciones científicas. Esta generalización viene acompañada de la emergencia de ideas afines en otros sistemas sociales.

Lo anterior nos conduce a analizar cómo ha evolucionado el constructivismo hasta llegar a una relativa estabilización en la sociedad contemporánea. Esta dimensión temporal lleva hacia la teoría de la evolución y hacia mecanismos de transformación de la estructura en sistemas sociales. Valiéndonos de estos recursos se puede establecer qué estructuras han derivado en un momento del tiempo, bajo el principio que la producción del sistema autopoiético no es realizable a discreción de éste, sino que debe afirmarse en un entorno del que es autónomo (Luhmann, 1998: 203). Es así como otros sistemas encuentran en la comunicación científica referencias constructivistas válidas y disponibles para la generación y operación de sus propias estructuras. Consideramos, de modo hipotético, que existen condiciones de complejidad social que probabilizan la instalación del constructivismo a nivel transversal en diversos sistemas sociales.

En la teoría de Luhmann (2007: 325-470), la evolución socio-cultural se produce a través de mecanismos de variación, selección y re-estabilización, de carácter probable, pues en cada paso cabe la posibilidad de rechazo y discontinuidad del proceso.

Revista Mad. N²3, Septiembre de 2010. Departamento de Antropología. Universidad de Chile http://www.revistamad.uchile.cl/23/blanco_06.pdf 


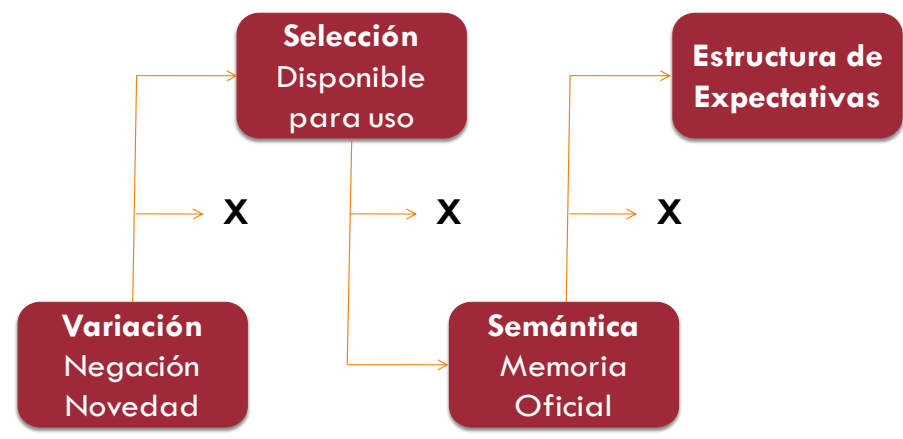

Figura 1 - Proceso de evolución socio-cultural (elaboración propia)

El primer momento supone una estabilización semántica previa. A partir de una comunicación inesperada -que rechaza contenidos de comunicaciones disponiblessurge una novedad posible de ser seleccionada. Hay variación en la medida en que esta comunicación sorpresiva produce diferencia, esto es, cuando se da una reproducción desviante a través de los elementos del sistema. Una variación no es automáticamente seleccionada. Múltiples variaciones pueden contradecir las comunicaciones vigentes, sin una reproducción en comunicaciones posteriores. Como lo expresa el mismo Luhmann, "la variación, entonces, no es génesis espontánea de lo nuevo [...] sino producción divergente de elementos del sistema" (2007: 364).

El segundo momento del proceso evolutivo es la selección. Ésta aparece cuando se apela a una comunicación desviante que, por su valor prometido, puede ser reutilizada en nuevas ocasiones de comunicación, es decir, "forman estructuras idóneas para el uso repetido, capaces de construir y condensar expectativas" (Luhmann, 2007: 358). En caso de darse la selección, la variación se elige entre las preferencias de sentido. La selección está referida a las estructuras del sistema, o sea, a las expectativas que guían la comunicación.

La re-estabilización es el tercer momento del ciclo evolutivo según Luhmann. Se da siempre y cuando los elementos seleccionados por la estructura logran el efecto de construir estructuras y condensarse en expectativas. Según el autor, "el concepto de re-estabilización designa secuencias de incorporación de cambios estructurales en un sistema que opera determinado por las estructuras" (Luhmann, 2007: 385). La reestabilización resulta, por lo tanto, de la aparición de variaciones seleccionadas como guías estables para comunicaciones ulteriores.

El proceso evolutivo -en tanto progresiones continuas de variación, selección y reestabilización- encuentra en el desarrollo semántico de los sistemas sociales un escenario para la observación empírica. En la medida que se dé cuenta de selecciones sociales afines al constructivismo, será posible respaldar la tesis de que el constructivismo aparece no sólo como una variación en el sistema social de la ciencia, sino que puede ser objeto de progresivas selecciones y convertirse en una distinción de observación disponible para hacer descripciones desde distintos sistemas. Con Luhmann, creemos que "nuestro problema inicial es saber si esta semántica es apropiada como auto-descripción de la sociedad, apta para la vida cotidiana y operativa" (Luhmann, 1998: 385). 


\section{Variaciones y selecciones sociales del constructivismo}

La epistemología constructivista asume como principio rector el carácter construido de la experiencia. Sostiene que el conocimiento que se posee de un objeto no se basa en correspondencias con algo externo, sino que es el resultado emergente de la construcción de un observador, en tanto realiza operaciones de distinción. En consecuencia, la validez de las explicaciones científicas, no dependerán de su conexión con un realidad externa, sino de un criterio de validez que, en el ámbito cognitivo de la ciencia, genera nuevas proposiciones presentadas como reformulaciones del fenómeno a explicar (Maturana, 1995).

Ahora bien, la aparición y desarrollo de proposiciones constructivistas en el seno de la ciencia no la circunscribe a ella. La formación de estructuras en el sistema ciencia, que asumen de una u otra manera el cuestionamiento a una realidad tal y como se presenta, tiene una evolución análoga en el pensamiento filosófico. Se sugiere por lo tanto que intuiciones de estas características están presentes en distintos sistemas de pensamiento a lo largo de la deriva filosófica; ideas que, en general, fungen como variaciones que no siempre son seleccionadas en desarrollos posteriores.

Expuestas las consideraciones científico-filosóficas de la evolución constructivista, se tematiza su gradual aparición en el ámbito de la vida cotidiana. La afirmación busca mostrar que la re-estabilización de perspectivas que incorporan elementos constructivistas puede hoy en día observarse en las expectativas que guían buena parte de la industria cultural de masas -favorecida por el desarrollo de nuevas tecnologías- sirviendo así de referencia disponible para nuevas comunicaciones.

\section{a. En las concepciones científicas}

La intuición de la influencia del observador en la descripción de la realidad genera todo un desarrollo científico con el objetivo de precisar las condiciones epistemológicas desde las que opera la ciencia. Cuestionando el realismo científico, desde principios del siglo pasado, comienzan a tomar fuerza interpretaciones que relativizan la composición ontológica de los fenómenos en distintos campos, abriendo camino para la emergencia de visiones constructivistas en el sistema de la ciencia. Estas variaciones van seleccionándose en ámbitos diversos, promoviendo la integración de nociones constructivistas en el discurso científico. Progresivamente, las selecciones se condensan y permiten su re-utilización en otros contextos científicos.

Desde el cuestionamiento al determinismo cartesiano-newtoniano que postula el principio de incertidumbre de Heisenberg -formulado en 1927- se pone en tela de juicio la obtención de datos estables de la realidad, los cuales sólo pueden entenderse en términos de probabilidad de ocurrencia. Por otra parte, el principio de incompletitud de Gödel también socava las bases de un positivismo acrítico, evidenciando que todo sistema formal incorpora proposiciones no demostrables ni refutables dentro de sí mismo (Vivanco, 2010). Estos dos descubrimientos -en la física y en la lógica matemática- contribuyen a la consolidación de la idea desviante de un acceso restringido a la realidad por parte de los observadores. En el caso de la física, la teoría de la relatividad y la física cuántica proveen intuiciones similares, que terminan por permear -por variación y selección- otros ámbitos de la ciencia.

Ya en la segunda mitad del siglo XX, surge en la biología la noción de Maturana y Varela, cuyo trabajo permitió sostener que "el sistema nervioso sólo observa los 
estados cambiantes del organismo del que forma parte y para cuya explicación dieron lugar a la teoría de la autopoiesis" (Arnold y Robles, 2004: 28). En una vía paralela, Von Foester reconsidera el principio de codificación indiferenciada de Müller, según el cual los sistemas sólo perciben magnitudes $y$, a partir de ellas, reconstruyen los estímulos en base a sus operaciones internas.

En la misma línea de incorporación del observador en las descripciones científicas contribuyen otras variaciones como: la lógica de Spencer-Brown, la cibernética de segundo orden de Maruyama y la teoría de la observación de segundo orden desarrollada por Luhmann. Estas concepciones constituyen posiciones epistemológicas en que la realidad deja de aparecer como dada y se entiende como una construcción del observador. Al respecto de estas selecciones progresivas, Luhmann aclara que "especialmente para la ciencia, es significativo ( $y$ desde hace mucho familiar) que se ha impuesto el entendido de la dependencia teórica y metodológica de todas las determinaciones acerca de la verdad. También podría hablarse de constructivismo o de que la referencia al mundo externo es sustituida o por lo menos mediatizada por la referencia a los condicionamientos de enunciados acerca del mundo externo" (Luhmann, 2006: 278).

\section{b. En el devenir filosófico}

Así como las auto-descripciones científicas de cuño constructivista han logrado erosionar, en cierta medida, las concepciones ontológicas registradas en el interior del sistema científico, el devenir filosófico configurado en occidente ha dado cuenta -en un marco cronológico evidentemente más extenso- de la emergencia de aquellas categorías epistemológicas que fundamentan el planteamiento constructivista. La inclusión de éste ámbito, más allá de propiciar una especie de legitimación histórica del constructivismo en la contemporaneidad, nos permitirá rastrear algunas de las formulaciones filosóficas que constituyen -a nuestro entender- variaciones que han probabilizado la selección de intuiciones cercanas al constructivismo a través de la historia del pensamiento filosófico.

Una primera intuición en el desarrollo filosófico se remonta a Platón (427-347 AC), quien expone -en palabras de Protágoras- la famosa frase "el hombre es la medida de todas las cosas". Asimismo, también en la visión de Sócrates pueden encontrarse cuestionamientos al conocimiento, en el entendido que la alegoría de la caverna refiere a la mediación sensible en el acceso al conocimiento. Alcanzar "lo verdadero" (las ideas), sólo es posible a partir de la razón. Esta visión sustancialista se mantiene relativamente estable durante la edad media y hasta Descartes, con la idea de la "duda metódica" cuya aplicación sobre los supuestos de conocimientos previos permite consolidar un principio firme y seguro para la ciencia de carácter racional. No se cuestiona el esencialismo, pero sí se entiende que los sentidos pueden conducir a engaños.

Desde la segunda mitad del siglo XVII, una serie de propuestas filosóficas desviantes, cercanas a las intuiciones del constructivismo, emergen y favorecen la selección de las mismas en otros ámbitos. Un hito importante fue la incorporación de la idea de la producción humana como límite del conocimiento de Vico. Su conocida sentencia verum ipsum factum (lo verdadero corresponde con lo hecho) reconoce la correspondencia de la verdad con el hecho y no con una realidad previa a la producción. La idea de Berkeley plasmada en la famosa frase esse est percipi (ser es ser percibido) es una variación en la misma línea: todo lo que puede conocerse es la percepción de un objeto. En este mismo contexto, la distinción -tanto de Locke como 
de Hume- entre ideas simples y complejas también refleja la emergencia de una variación significativa. El corolario de esta distinción es que no hay conocimiento más allá de las percepciones simples o átomos de percepción, las cuales se conjugan para construir las ideas complejas. Estas perspectivas desviantes, salvo quizás la de Vico, hasta ese momento seguían orientándose desde concepciones esencialistas.

En un plano más contemporáneo, el idealismo alemán logró precisamente poner en tela de juicio los esencialismos, punto no resuelto por la filosofía precedente. Kan propuso que la realidad se construye a través de las categorías del pensamiento. Según Luhmann (2007) es en Kant donde podemos encontrar un punto de inflexión, ya que logra solucionar el problema del conocimiento en el ámbito exclusivo de la subjetividad. Es decir, "en la relación del sentido interior con las representaciones del entendimiento $-y$ no en la relación del sujeto con el mundo exterior" (p. 690). Asimismo, se consigue desplazar el "problema desde la dimensión objetual (correspondencia) a la dimensión temporal" (p. 690). El esquematismo -elemento central de esta conceptualización referida al sujeto- brinda una explicación procedimental que no copia o retrata el 'objeto', sino que, por el contrario, lo construye. De todos modos, la perspectiva kantiana no está exenta de dificultades ya que, en palabras de Luhmann, el sujeto sería "irreflexivo con respecto a las distinciones primarias a las cuales debe la posibilidad de observar" (p. 690).

El esencialismo encuentra uno de sus más grandes críticos en Hegel, quien finalmente reconoce que nada es susceptible de conocer más allá del pensamiento. En ese sentido, Hegel (1966: 104) finalmente señala que el pensamiento constituye el límite del conocimiento que "se alza pues el telón sobre lo interior (del objeto, ahí esta entonces lo que debería verse, porque es la esencia), y lo presente es el acto por el cual lo interior (del sujeto) mira lo interior (del objeto), y lo que ve es que tras el Ilamado telón que debía cubrir el interior, no hay nada que ver. A no ser que se ponga uno mismo en ese lugar, tanto para ver, como para que haya algo que sea visto".

Las perspectivas presentadas, podrían considerarse como antecedentes a las formulaciones constructivistas contemporáneas, como variaciones que contaron con niveles limitados de selección. Constituyen intuiciones contrarias a la posibilidad acrítica de un acceso directo a la realidad, ya sea por una vía empírica o por una vía racional. Con las contribuciones subjetivistas y anti-esencialistas del idealismo alemán, los fundamentos del constructivismo ya habían sido enunciados, lo que había fomentado su selección en comunicaciones filosóficas futuras. Probablemente, el denominado giro lingüístico en la filosofía de mediados del siglo $\mathrm{XX}$, allanó el camino para la emergencia y consolidación del constructivismo contemporáneo.

\section{c. En la vida cotidiana}

En las producciones culturales -particularmente en el cine y aquellas que se transmiten por los medios de difusión- es posible encontrar una serie de intuiciones asociadas al constructivismo. Una de las más famosas metáforas es la escena de la película Matrix, en la que el protagonista -Neo (Keanu Reeves)- descubre, en diálogo con un niño, que la realidad depende de la percepción que él tenga de la misma.

- No trates de doblar la cuchara, eso es imposible. En vez de eso, sólo trata de darte cuenta de la verdad.

- ¿Qué verdad?

- No hay ninguna cuchara.

- ¿No hay ninguna cuchara?

- Entonces verás que no es la cuchara lo que se dobla. Eres sólo tú mismo. 
Interpretando de manera constructivista el mensaje de los hermanos Wachowski, podemos inducir que para doblar la cuchara es necesario darse cuenta de que "no hay una cuchara". Para poder observar la realidad, es necesario darse cuenta de que no hay realidad". O dicho de otro modo -no tan radicalmente- "hay algo que doblar u observar", pero no es ni la cuchara ni la realidad, sino uno mismo: el observador. Aunque la escena no es exactamente coherente con la teoría constructivista de la observación, su cercanía semántica es evidente. Al contrario del caso de las teorías constructivistas en la ciencia y la filosofía -cultivadas sólo por especialistas- la película Matrix resulta ampliamente difundida en la cultura popular.

En términos evolutivos, distinguimos estos eventos como variaciones en las comunicaciones acerca de la "realidad" y su conocimiento. Suponemos que en la sociedad actual su difusión hace pensar que producciones culturales como Matrix constituyen variaciones con perspectivas de condensación en estructuras de expectativas. De hecho, en varias instancias de conversación cotidiana, el argumento de la película resulta familiar y está disponible para la comunicación, coordinando expectativas compartidas de sentido. Estas variaciones se presentan masivamente y con un alto grado de impacto en la sociedad. De cierta manera, podemos observar cómo -en el contexto global- una perspectiva epistemológica como la constructivista puede ser comunicada por los medios de difusión y seleccionada por amplios sectores de la sociedad.

La introducción del constructivismo en lo cotidiano requiere que éste sea seleccionado y estabilizado como disposición estructurante de la sociedad. En ámbitos científicos, la divulgación de dicho paradigma puede entenderse como variaciones en las comunicaciones científicas (papers, investigaciones, ponencias, etc.), pero estas comunicaciones también se observan en manifestaciones de la cultura popular, a saber, el cine, la literatura, internet y series televisivas, entre otras fuentes. Seguimos a Luhmann cuando entendemos que la variación evolutiva "es un fenómeno tan general, amplio, y masivo, que no podría ser confiado a roles especiales. Los intelectuales pueden fungir como amplificadores; pero ellos mismos, a su vez, están a tal punto determinados por las modas, por las polémicas y por la consistencia semántica de sus representaciones, que no pueden poner a prueba una variación suficientemente abierta" (Luhmann, 1998: 219). En ese sentido, así como el sistema científico está atravesado por interacciones cotidianas, también la difusión del constructivismo puede observarse en espacios no científicos, a saber, en la industria cultural.

En estas comunicaciones -en la actualidad- es posible reconocer una semántica de tono constructivista: no sólo se sospecha de la realidad, sino también se abre la posibilidad de la creación de la realidad misma. La pregunta por el acceso a la realidad, por el conocimiento, por el control de nuestras vidas y el libre arbitrio, por la validez de distintas perspectivas, se hacen disponibles como variaciones en la comunicación cotidiana, proceso que se probabiliza con los medios de difusión.

El que esta semántica sea seleccionada, pasa hoy en día -en gran medida- por el lugar que ocupan en la sociedad las tecnologías de la información y las comunicaciones (TIC). Es así como celulares, computadores, satélites, consolas de videojuegos, reproductores de música y cámaras digitales, entre otros ejemplos, favorecen -por medio de la difusión- la selección de semánticas cercanas al constructivismo. De cierta manera, el uso cotidiano y cada vez más naturalizado y transparente de estas 
tecnologías (Zizek, 2006) confirman la sospecha que desde el cine se comunica, haciendo más probable la selección de variaciones sobre qué es real.

Como segundo modo de probabilizar la selección de semánticas constructivistas, el uso masificado de estas tecnologías y el cambio evidente en las formas de comunicación y organización de la vida operan como sustento para suponer que el conocimiento de la realidad es ambiguo, o al menos, no universal. Entonces, ¿se estará estabilizando socialmente una forma de reconocimiento constructivista, nuevas premisas que favorecen el cuestionamiento de las premisas? El concepto de "realidad virtual" parece representar, por un lado la posibilidad de construcción de realidades con efectos reales, en un escenario virtual. Asimismo, cuestiona la suposición de que existe algo real en contraposición a lo virtual y, por otra parte, genera la conciencia de que lo real puede ser tan virtual como lo virtual. Son recurrentes a las discusiones cotidianas sobre el problema de la realidad de lo virtual y la virtualidad de lo real, que aparece tematizada frecuentemente en fenómenos como los reality shows, el cibercrimen, el cine 3D y otras formas culturales.

Además de las producciones culturales y la construcción de una realidad virtual a través de las TIC, la globalización favorece el reconocimiento de la coexistencia de múltiples culturas en la sociedad contemporánea. Ello -en vista del encuentro directo entre diferentes culturas- genera como condición de comunicación la consideración de cosmovisiones diversas como válidas. Lo multicultural, lo pluricultural, lo transcultural, lo intercultural, son conceptos cada vez más comunes, y que dan cuenta de una selección comunicativa que reconoce distintas observaciones de mundo. Lo anterior también reafirma la tesis de que se estaría estabilizando de modo masivo una forma semántica cercana al constructivismo.

La instalación del constructivismo en la vida cotidiana encuentra evidencia en una serie de variaciones cercanas a tal perspectiva epistemológica, que comienzan a ser seleccionadas y probabilizan nuevas comunicaciones. Por una parte, películas como Matrix introducen la temática de la incertidumbre de lo real en las comunicaciones cotidianas, cuya selección se hace más probable por la expansión de los medios de difusión y las TIC. La instalación del concepto de realidad virtual y el contexto multicultural de comunicación que permiten las tecnologías como internet y el cine, estarían promoviendo la selección masiva de formas culturales afines al constructivismo.

\section{d. Perspectivas de estabilización}

En base a la constatación de que en la sociedad "no sólo ocurren cambios, sino también se observan, es decir, se comunica que ocurren" (Luhmann, 2007: 450), la propuesta central de este trabajo ha sido describir las condiciones evolutivas del constructivismo en la sociedad contemporánea. Consideramos que estamos siendo testigos de comunicaciones sobre cambios operativos en la sociedad, y la instalación del constructivismo respondería a una selección susceptible de integrarse a la estructura semántica y probabilizar así nuevas comunicaciones. El constructivismo sería, desde esta perspectiva, una adquisición evolutiva.

Sin embargo, es necesario preguntarse por las condiciones sociales para la estabilización del constructivismo. En la película "The Truman Show" de Peter Weir, la escena final ilustra bien esta situación: Truman, un sujeto que ha permanecido toda su vida en una ciudad-estudio como protagonista de un show de televisión sin saberlo, se enfrenta -en el desenlace- a la decisión de salir de Seaheaven hacia el mundo real o 
bien, intentar olvidar que ha estado viviendo en una ilusión para volver a la comodidad del mundo que han creado para él.

La sociedad contemporánea se encuentra en una situación análoga respecto del constructivismo: o bien integra al constructivismo a la estructura de expectativas o simplemente lo olvida sin consecuencias estructurales. Esta situación conduce, por una parte a la discusión sobre la reversibilidad de la evolución sociocultural y, por otra, a la interpretación del constructivismo como adquisición evolutiva de la sociedad contemporánea. Respecto de la irreversibilidad de la evolución, Luhmann reconoce que "las estructuras detienen el tiempo de manera reversible, puesto que mantienen abierto un repertorio limitado de posibilidades de selección [...] Los procesos, por su lado, marcan la irreversibilidad del tiempo; consisten en acontecimientos irreversibles. No pueden ir hacia atrás" (Luhmann, 1998: 65). En cuanto al segundo punto cabe cuestionarnos, ¿en qué medida es posible interpretar la aparición de elementos constructivistas como una "adquisición evolutiva" de la sociedad contemporánea?

Las indicaciones desarrolladas a lo largo del trabajo, así como las evidencias que pretenden ilustrar estos argumentos, llevan a sugerir que la observación del constructivismo desde el punto de vista de su dimensión temporal refleja un proceso de gradual selección y tentativa estabilización en la actualidad. ¿Cómo ocurre esto?

Tomando como punto de partida investigaciones sobre semánticas históricas, Reinhart Koselleck ha observado cómo es que los conceptos sociales "contienen una concreta pretensión de generalidad y son siempre polisémicos" (Koselleck, 1993: 116). Al reconstruir el proceso de emergencia y estabilización de una semántica del constructivismo queda manifiesta la multiplicidad de elementos que son subsumidos en su campo de significación, así como el carácter polémico que este asume. Esto es así, como se ha visto, en el sistema científico, en la historia del pensamiento filosófico y virtualmente- en las referencias de la vida cotidiana. $Y$ sin embargo comunicaciones constructivistas no son exitosas sino hasta que el concepto logra desplegar sus "posibilidades estructurales" (Koselleck, 1993: 123).

En efecto, sugerimos que la re-estabilización de comunicaciones constructivistas puede ser entendida como una "adquisición evolutiva". Luhmann es taxativo al señalar que todo resultado de la evolución posibilita "una complejidad más alta" (Luhmann, 2007: 399). A nivel de las estructuras del sistema, esto dice relación con cómo ciertas apariciones llegan a verse como más compatibles para el establecimiento de relaciones complejas en los sistemas. Sea a través de la crítica al positivismo en el marco de la ciencia, la desontología que ensayan distintos sistemas filosóficos, o el aún pedestre cuestionamiento a una realidad cotidiana asumida como estática e incuestionable, los elementos constructivistas apoyan la conexión de nuevas referencias $y$, en consecuencia, la multiplicación de posibilidades. Con todo, la idoneidad de una alternativa como esta sólo debe verse en el marco funcional de un conjunto de soluciones equivalentes, que pueden o no actualizarse respecto a la delimitación de un problema determinado.

De ser plausible las indicaciones anteriores, queda todavía por establecer si estas comunicaciones constructivistas representan una "ventaja evolutiva" en la deriva social. Sobre este punto, Luhmann ha señalado que "las adquisiciones evolutivas eligen las reducciones de forma tal que pueden ser compatibles con una complejidad más alta y con frecuencia (y la mayoría de las veces gradualmente) es lo único que las hace posibles" (Luhmann, 2007: 400-401). Se identifica, por tanto, que el constructivismo suscita un valor evolutivo siempre y cuando se ponga en relación a la creciente 
complejidad social. Esto no equivale, por supuesto, a un principio teleológico o una defensa normativa del concepto, sino a una relación funcional en una selección que es contingente, es decir, que no es necesaria ni imposible.

Es razonable suponer que en torno a adquisiciones evolutivas como el constructivismo ciertas estructuras hayan ganado viabilidad. Hemos relevado esta hipótesis a partir de la descripción del mecanismo de variación, selección y re-estabilización del constructivismo en la evolución social, indicando su valor semántico en el sistema ciencia, el pensamiento filosófico y la industria cultural moderna. Ahora bien, esto abre la posibilidad de que referencias constructivistas hayan logrado condensarse en estructuras de expectativas en el orden de los sistemas, esto es, "condiciones que delimitan el ámbito de las operaciones capaces de empalmarse" (Luhmann, 1998: 202).

La orientación de hipótesis como estas sirve de marco para nuevas interrogaciones acerca del valor del constructivismo desde el punto de vista del manejo de la complejidad social en múltiples sistemas y desde distintos problemas de referencia. Como cualquier construcción, ésta puede volverse obsoleto en la medida que se recurra a otras vías para la realización de empalmes comunicativos. A nivel estructural, el constructivismo puede ser olvidado; a nivel operativo siempre expone a las estructuras sociales a la evolución. RM

\section{Referencias}

Arnold, M. (2004). Introducción a las Epistemologías Sistémico/ Constructivistas. En F. Osorio (Editor), Ensayos sobre Socioautopoiesis y Epistemología Constructivista (pp. 715). Santiago de Chile: Facultad Ciencias Sociales, Universidad de Chile.

Arnold, M. (2006). Fundamentos de la observación de segundo orden. En M. Canales (Coordinador), Metodologías de investigación social. Introducción a los oficios (321348). Santiago de Chile: Lom.

Arnold, M. \& Robles, F. (2004). Explorando caminos transilustrados más allá del neopositivismo: epistemologías para el siglo XXI. En F. Osorio (Editor), Ensayos sobre Socioautopoiesis y Epistemología Constructivista (pp.26-45). Santiago de Chile: Facultad de Ciencias Sociales, Universidad de Chile.

Haines, V. (2007). Evolutionary explanations. En S. Turner \& M. Risjord (Editors). Philosophy of Anthropology and Sociology. New York: Elsevier.

Hegel, G. F. W. (1966). Fenomenología del espíritu. México, D. F.: Editorial Fondo de Cultura Económica.

Koselleck, R. (1993). Futuro pasado: para una semántica de los tiempos históricos. Barcelona: Paidós.

Luhmann, N. (1998). Sistemas sociales: lineamientos para una teoría general. Barcelona: Anthropos.

Luhmann, N. (2006). Sociología del riesgo. México, D. F.: Universidad Iberoamericana.

Luhmann, N. (2007). La sociedad de la sociedad. México, D. F.: Herder. 
Mascareño, A. (2008). Acción, estructura y emergencia en la teoría sociológica. Revista de Sociología, Universidad de Chile, (22), 217-256.

Maturana, H. (1995). La ciencia y la vida cotidiana: la ontología de las explicaciones científicas. En P. Watzlawick \& P. Krieg (Compiladores), El ojo del observador: contribuciones al constructivismo (pp. 157-194). Barcelona: Gedisa.

Razeto-Barry, P. y Cienfuegos, J. (2008). La paradoja de la probabilidad de lo improbable y el pensamiento evolutivo de Niklas Luhmann. Ponencia presentada en el seminario Niklas Luhmann, a diez años. Santiago de Chile.

Vivanco, M. (2010). Sociedad y complejidad. Del Discurso al modelo. Santiago: Lom.

Zizek, S. (2006). Lacrimae rerum. Ensayos sobre cine moderno y ciberespacio. Madrid: Debate. 